\title{
TRAMPAS DE LUZ CON PANEL PEGANTE PARA LA CAPTURA DE ADULTOS DE Prodiplosis longifila Gagné (Diptera: Cecidomyiidae) EN EL CULTIVO DE ESPÁRRAGO
}

\section{LIGHT TRAPS WITH GLUE PANELS TO CAPTURE Prodiplosis longifila Gagné (Diptera: Cecidomyiidae) ADULTS IN AN ASPARAGUS CROP}

\author{
Fredy Camborda Z. ${ }^{\text {, Jorge Castillo V. }{ }^{2} \text { y Susana Rodríguez Q. }}{ }^{3}$
}

\begin{abstract}
Resumen
Se determinó el efecto de trampas de luz con panel pegante con cuatro diferentes tipos de luz: blanca, negra, amarilla incandescente y amarilla de lámpara a querosene en la captura de adultos de la "mosquilla de los brotes" Prodiplosis longifila Gané. El experimento se realizó en el valle de Supe (Lima), en un campo comercial de espárrago verde de la variedad Ida Lea F1, con presencia de larvas y adultos de $P$. longifila. Se evaluó el número de adultos capturados por trampa de luz con panel pegante de $7.5 \mathrm{~m}^{2}$ de área entre las 6 pm y 6 am, durante los meses de marzo y abril del 2008.

Los resultados muestran que el número promedio de adultos de $P$. longifila capturados con la lámpara fluorescente de luz blanca fue de 134012 individuos, con la lámpara fluorescente de luz negra fue de 107607 individuos, con la lámpara de luz incandescente amarilla fue de 65107 individuos, y con la lámpara a querosene de luz amarilla con 4893 individuos capturados. Las trampas con lámparas de luz blanca y de luz negra fueron las más eficientes en número de adultos capturados por panel pegante.

Palabras clave: Prodiplosis longifila, trampa de luz con panel pegante, luz negra, luz blanca, luz incandescente amarilla, luz amarilla de lámpara a querosene.
\end{abstract}

\begin{abstract}
The effect of light traps with glue panel with four different types of light: white, black, yellow incandescent and yellow kerosene lamp on Prodiplosis longifila Gané adults' captures was determined. The essay was performed at Supe Valley (Lima), in a commercial field of green asparagus Ida Lea F1 variety, with conspicuous presence of larvae and adults of $P$. longifila. The number of adults captured with sticky-light traps of $7.5 \mathrm{~m}^{2}$ from $6 \mathrm{pm}$ to $6 \mathrm{am}$, from March to April, 2008, were registered.

The results show that the average number of adults of $P$. longifila captured with white light fluorescent lamp traps was 134012 individuals, with black light fluorescent lamp traps, 107607 individuals, with the incandescent yellow light lamp traps, 65107 individuals, and with the yellow light kerosene lamp traps, 4893 individuals. White light and black light lamp traps were more efficient on $P$. longifila adults' captures.

Key words: Prodiplosis longifila, sticky-light trap, black light, white light, incandescent yellow light, yellow light kerosene lamp.
\end{abstract}

\section{Introducción.}

El espárrago es una planta originaria de climas templados del mediterráneo europeo que ha logrado adaptarse eficientemente a los climas subtropicales de la costa peruana alcanzando altos rendimientos y altos estándares de calidad e inocuidad, posicionando al Perú como uno de los primeros exportadores de espárrago en el mundo el año 2014.

En los agroecosistemas del cultivo de espárrago en la costa central y costa norte del Perú, la "mosquilla de los brotes” $P$. longifila, es considerada como la plaga clave o principal del espárrago; este insecto perjudica a la planta durante todo su desarrollo fenológico, principalmente durante la brotación, ramificación y apertura de filocladios, lo cual provoca que en muchos casos, las cosechas se realicen después del primer brote. Así también, debido a los fuertes daños causados por esta plaga, el uso de plaguicidas químicos dirigidos al control de larvas también se ha incrementado notablemente, trayendo consigo la selección de poblaciones cada vez más resistentes a los diferentes plaguicidas usados (Castillo, 2006). 


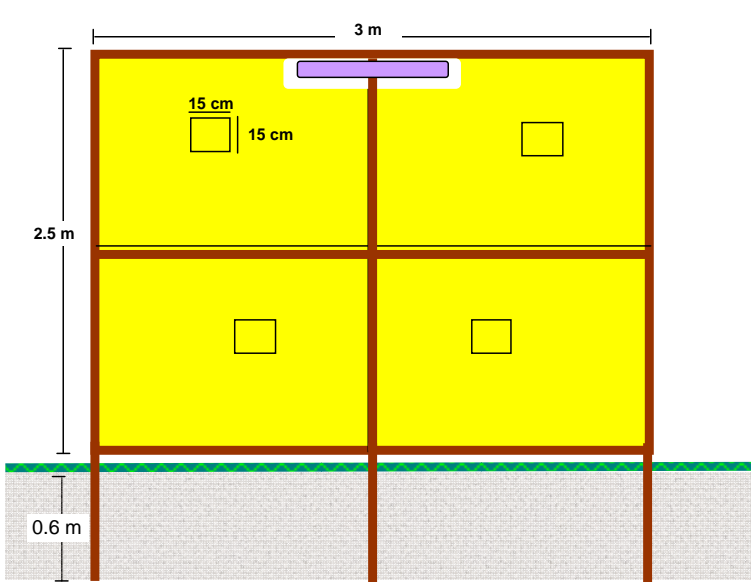

Leyenda:

Ubicación de la luz

Parantes de madera

Plástico pegante color amarillo

Superficie del suelo

Profundidad del parante

Área evaluada

Figura 1. Esquema de trampa de luz con panel pegante.

Fuente: Elaboración propia (2008).

Actualmente, el manejo de $P$. longifila se está orientando al control de los adultos para evitar que las poblaciones se incrementen en el cultivo (García, 2006), por lo que dentro de un Programa de Manejo Integrado de Plagas (MIP), el uso de las trampas de luz con panel pegante es una de las principales medidas para la captura y mortalidad de adultos, la cual puede emplearse no solo con fines de control, sino también para programas de seguimiento, detección y evaluación de la plaga.

El uso de las trampas de luz con panel pegante, viene a ser uno de los principales componentes del MIP en el cultivo de espárrago y de otros cultivos de importancia económica en los que la "mosquilla de los brotes" se comporta como plaga clave; sin embargo, aún no se ha demostrado a nivel experimental bajo condiciones de la costa central, la eficiencia de las trampas de luz con panel pegante en la captura de los adultos de $P$. longifila.

La presente investigación determina el efecto de las trampas de luz con panel pegante con cuatro diferentes tipos de luz para la respectiva atracción y captura de adultos de $P$. longifila en el cultivo de espárrago verde.

\section{Materiales y métodos.}

El experimento se realizó en un campo comercial de espárrago verde de la variedad Ida Lea F1, ubicado en la parte media del valle de Supe, a 170 km al norte de la ciudad de Lima- Perú. Durante el desarrollo del experimento, las condiciones climáticas promedio en el valle fueron: temperatura máxima: $31.5^{\circ} \mathrm{C}$, temperatura mínima: $20.9^{\circ} \mathrm{C}$, humedad relativa máxima: $80.1 \%$ y humedad relativa mínima: 58.6\%; correspondiendo a una estación típica de fines de verano e inicios de otoño.

El experimento se realizó durante la quinta campaña de producción de un campo comercial de espárrago verde de tres años de instalado (2004) en un área neta de 6 hectáreas en pleno crecimiento vegetativo. El campo experimental estuvo delimitado por el norte, con un campo de camote; por el sur con un campo de caña de azúcar y otro de yuca; por el oeste con un campo de alcachofa, y por el este con campos de maíz y camote.

Las trampas de luz con panel pegante fueron diseñadas con parantes de madera, siendo las dimensiones del marco de $3 \mathrm{~m}$ de ancho por $2.5 \mathrm{~m}$ de largo; los parantes fueron enterrados a una profundidad de $0.6 \mathrm{~m}$. El panel de captura estuvo comprendido por un área neta de $7.5 \mathrm{~m}^{2}$ de plástico amarillo cubierto con una sustancia pegajosa, aceite de motor SAE 50, de menor costo, mayor duración, y mayor eficiencia de captura (Mujica et al., 2000).

En cada trampa se ubicó la fuente de atracción luminosa en la parte central superior. Para el caso de los tratamientos con luz blanca y luz negra los tubos fluorescentes fueron ubicados horizontalmente, mientras que en los casos de los tratamientos de luz amarilla incandescente de bombilla y luz amarilla de lámpara a querosene, fueron ubicados en forma vertical, según Figuras 1, 2, 3, 4 y 5.

La parcela seleccionada presentaba daños visibles de $P$. longifila, y en ella se habían realizado dos aplicaciones de insecticida en la campaña, estaba ubicada a una distancia aproximada de $2 \mathrm{~km}$ de la población más cercana: Centro Poblado de la Campiña de Supe.

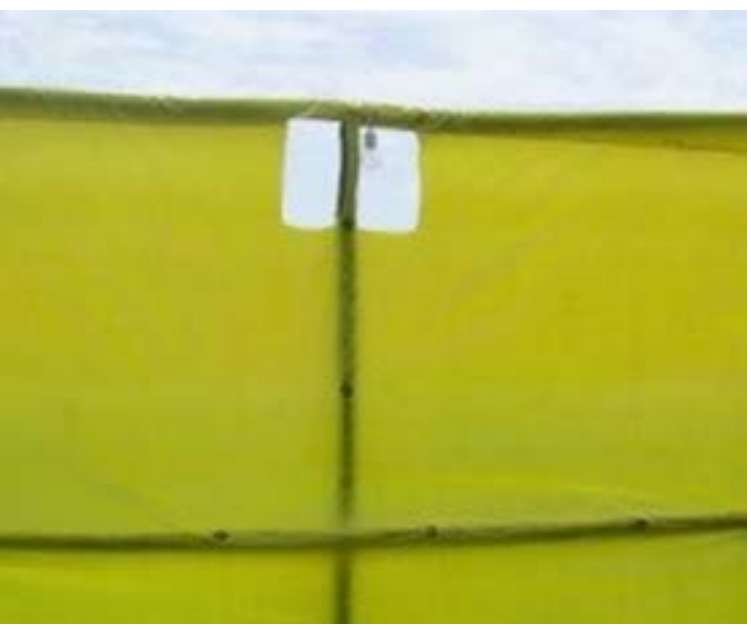

Figura 2. Lámpara de luz amarilla incandescente, de bombilla. 


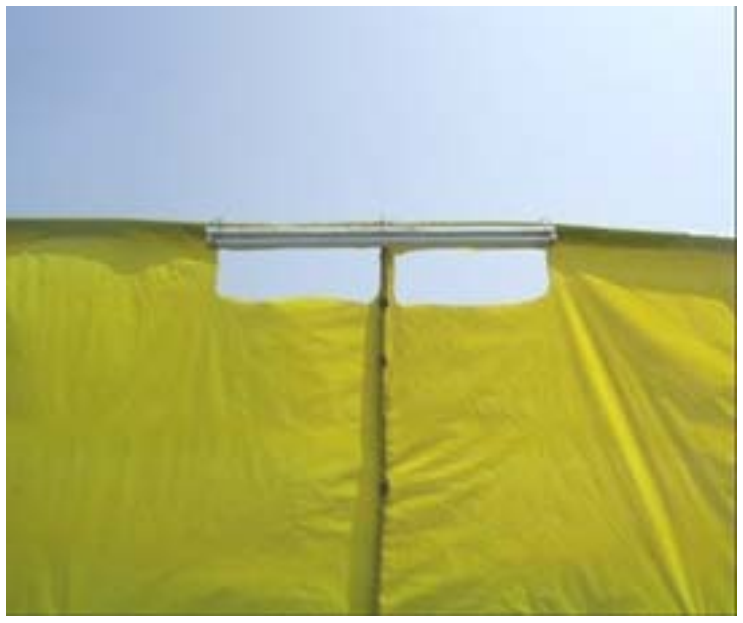

Figura 3. Lámpara de luz blanca fluorescente.

Dentro del campo experimental se ubicaron cuatro puntos equidistantes de 122.45 metros de distancia entre punto, aproximadamente; se instaló en cada punto una trampa de luz con panel pegante, permaneciendo fija en el punto de ubicación de los parantes de madera, cambiándose luego de posición aleatoriamente las cuatro trampas de luz.

Como fuente de energía para el funcionamiento de las lámparas eléctricas fluorescentes de luz blanca, luz negra y luz amarilla incandescente de bombilla, se usó un generador eléctrico de 2 tiempos, con 1000 watts de potencia; para el caso de la lámpara de luz amarilla que utilizan los regadores en campo la fuente de energía fue querosene. La alimentación de la energía eléctrica desde el generador eléctrico hacia las lámparas luminarias instaladas, se hizo a través de cables de luz $\mathrm{N}^{\circ} 14$. Los tratamientos instalados se describen en la Tabla 1.

Para comparar el número de adultos de $P$. longifila capturados en cada tratamiento, se realizó un análisis

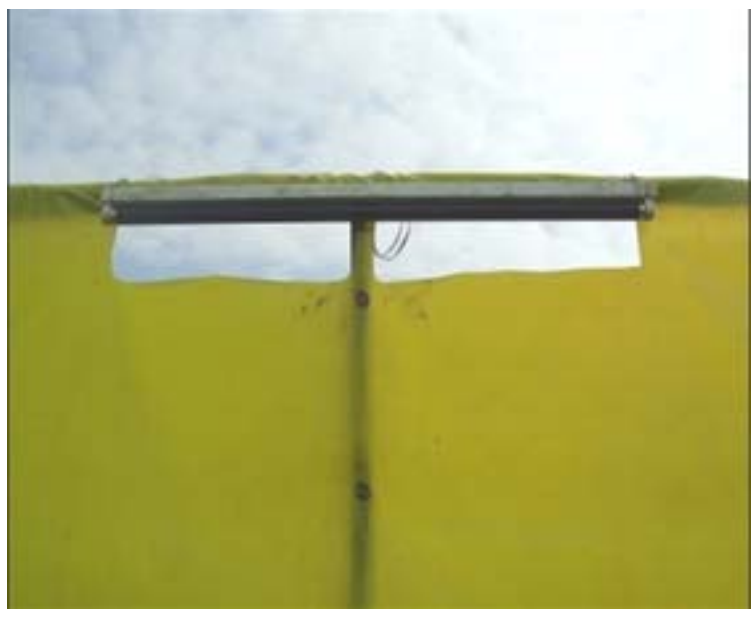

Figura 4. Lámpara de luz negra fluorescente.

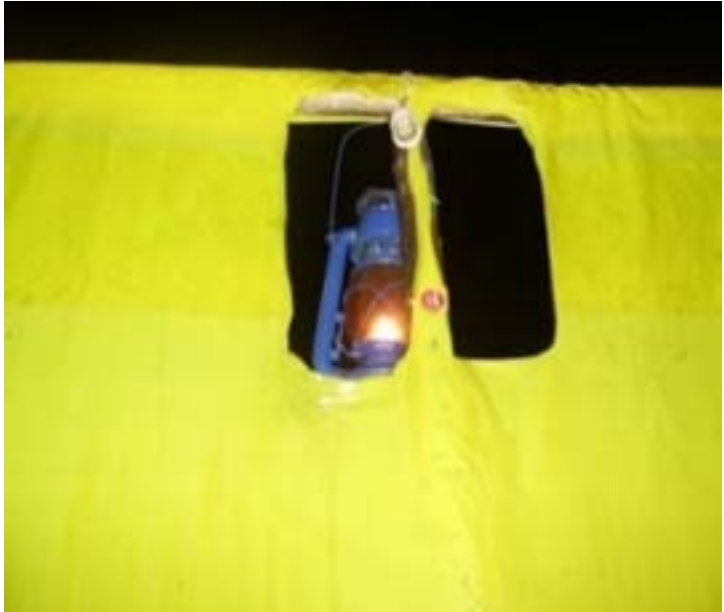

Figura 5. Lámpara de luz amarilla a querosene.

de variancia $(\mathrm{p}<0.05)$ para un diseño de bloques completos al azar (DBCA) con cuatro repeticiones por tratamiento. Para las comparaciones entre las medias de los tratamientos se utilizó la Prueba de Duncan a un nivel de significación de cinco por ciento, sin transformación de datos.

Las trampas de luz con panel pegante fueron prendidas durante todas las noches en las que se realizó el experimento, desde las 6 pm hasta las 6 am del día siguiente, evaluándose diariamente el parámetro: número total de adultos de $P$. longifila capturados por un lado del panel. Cada bloque correspondió al promedio de siete días consecutivos evaluados, luego se dejaron tres días sin evaluación, y luego de cambiar de posición aleatoriamente las cuatro trampas, se reanudaba la evaluación del siguiente bloque, hasta culminar la evaluación de los cuatro bloques estudiados, según Figura 6.

En cada trampa de luz con panel pegante se colocaron por cada lado del panel cuatro porciones de plástico amarillo $\left(15 \mathrm{~cm} \times 15 \mathrm{~cm}=225 \mathrm{~cm}^{2}\right)$ untados con aceite de motor SAE 50, y con la ayuda de una lupa de veinte aumentos se procedía a realizar el conteo de los adultos en las cuatro porciones a manera de muestras, obteniéndose luego el promedio de individuos capturados por noche en $(15 \mathrm{~cm} \times 15$ $\mathrm{cm}=225 \mathrm{~cm}^{2}$ ).

\section{Resultados.}

-Efecto de los diferentes tipos de luz con panel pegante en la captura de adultos de $P$. longifila

En la Tabla 2, se muestran los resultados del número promedio de adultos de $P$. longifila capturados por noche bajo el efecto de los cuatro tipos de luz usados en las trampas. El análisis de varianza muestra diferencias con significación estadística en el número de adultos de $P$. longifila capturados por las diferentes trampas de luz utilizadas. 
Tabla 1. Tratamientos de las trampas de luz con panel pegante y características de la lámpara de luz

\begin{tabular}{|c|c|}
\hline Tratamientos & Características de la lámpara de luz \\
\hline $\begin{array}{l}\text { T1: Trampa de luz } \\
\text { amarilla incandescente }\end{array}$ & $\begin{array}{l}\text { Bombilla de filamento de tungsteno, de } 40 \text { watts de potencia. La composición espectral } \\
\text { incluye la luz visible y principalmente infrarroja, producida por el filamento de tungsteno, } \\
\text { marca Philips. }\end{array}$ \\
\hline $\begin{array}{l}\text { T2: Trampa de luz } \\
\text { blanca fluorescente }\end{array}$ & $\begin{array}{l}\text { Tubo fluorescente de } 1200 \mathrm{~mm} \text { de longitud y } 38 \mathrm{~mm} \text { de ancho, de } 40 \text { watts de potencia. } \\
\text { Tono de luz: "luz día”. Distribución espectral principalmente en la región visible y en } \\
\text { menor grado en el ultravioleta cercano, marca Philips. }\end{array}$ \\
\hline $\begin{array}{l}\text { T3: Trampa de luz negra } \\
\text { fluorescente }\end{array}$ & $\begin{array}{l}\text { Tubo fluorescente de } 1200 \mathrm{~mm} \text { de longitud y } 38 \mathrm{~mm} \text { de ancho, de } 40 \text { watts de potencia. } \\
\text { Tipo BLB. La lámpara fluorescente de luz negra usa un fósforo especial y un vidrio filtrado } \\
\text { para que la mayor parte de la energía radiante esté dentro de la gama espectral de la luz } \\
\text { negra o casi ultravioleta, marca Philips. }\end{array}$ \\
\hline $\begin{array}{l}\text { T4: Trampa de luz } \\
\text { amarilla a querosene }\end{array}$ & $\begin{array}{l}\text { Lámpara artesanal de vidrio, también conocida como mechero. Usa como fuente de energía } \\
\text { querosene. La luz producida por esta lámpara tiene un mayor espectro en la luz infrarroja, } \\
\text { con menor rango en la luz visible, y muy poco o despreciable en la luz ultravioleta. }\end{array}$ \\
\hline
\end{tabular}

Para determinar la diferencia de captura de los adultos de $P$. longifila entre los cuatro tipos de luz usados en las trampas de luz pegante, se realizó la Prueba de Comparación Múltiple de Duncan a un nivel de significación de cinco por ciento, encontrándose que el mayor número promedio de adultos de $P$. longifila capturados por noche fue obtenido con las trampas de luz blanca fluorescente (T2) y con la luz negra fluorescente (T3), siendo ambos tratamientos estadísticamente similares entre sí.

La trampa de luz amarilla incandescente (T1) presenta estadísticamente un menor número de captura de adultos de $P$. longifila que la trampa de luz blanca fluorescente (T2), pero estadísticamente es similar a la trampa de luz negra fluorescente, para una misma potencia de 40 watts. La trampa de luz amarilla a querosene (T4) es el tratamiento que presenta el menor número promedio de adultos de $P$. longifila capturados por noche. Según se puede observar en la Tabla 3.

-Eficacia de captura de adultos de $P$. longifila

Los resultados obtenidos en el presente experimento, nos muestran que la eficacia de captura del adulto de la "mosquilla de los brotes” $P$. longifila depende mucho de la atracción en menor o mayor grado hacia diferentes longitudes de onda de luz artificial. El insecto muestra mayor atracción hacia la trampa de luz blanca fluorescente, seguido de la trampa de luz negra fluorescente, y en último lugar la trampa de luz amarilla incandescente.

\section{Discusión}

La capacidad de atracción que las lámparas eléctricas tienen para los

fotopositivos depende del rango de la radiación electromagnética de longitud de onda, la cantidad de energía emitida (energía radiante), la intensidad (brillantez), y del

\section{$\mathrm{CV}=2.55 \%$}

tamaño de la fuente de luz (Martín \& Woodcock, 1983, Nacional Academy of Sciences, 1985, Cisneros, 1995 y Carranza et al., 1995). Lo cual explica la diferencia en la capacidad de atracción de $P$. longifila hacia las lámparas eléctricas y de combustión, confirmando que la calidad de luz o tipo de luz influye considerablemente en el número y en la diversidad de insectos capturados, como lo consideran Southwood (1978), Walker \& Galbreath (1979), Nacional Academy of Sciences (1985), Vergara (2000), Gil (2008), Márquez (2005), Castillo (2006) y Weinzierl et al. (2008).

Las lámparas eléctricas producen longitudes de onda desde $180 \mathrm{~nm}$ hasta $5000 \mathrm{~nm}$, es decir, una porción muy pequeña del total del espectro de energía electromagnética. La porción del espectro producida por lámparas eléctricas se divide en tres regiones sin líneas agudas de demarcación, habiendo un cambio gradual de una región a otra. Estas regiones son: la ultravioleta (180 a $380 \mathrm{~nm})$, la visible (380 a $760 \mathrm{~nm})$ y la onda corta o casi infrarroja (760 a $5000 \mathrm{~nm}$ ). La región ultravioleta, que es invisible al ojo humano, se divide en: ultravioleta lejana, conocida como UV-C, germicida, distante, u onda corta de rayos UV, que oscila entre a 180 a $280 \mathrm{~nm}$; ultravioleta media, conocida como UV-B u onda mediana, que va desde 280 a $320 \mathrm{~nm}$; y la ultravioleta cercana, conocida como UV-A, luz negra, black light, onda larga de rayos UV, con longitud de onda de 320 a $380 \mathrm{~nm}$; esta luz negra está cerca al rango de UV como opuesto a la luz visible y brillante, y es el más atractivo a los insectos voladores. (Nacional Academy of Sciences, 1985).

Tabla 2. Análisis de varianza del número de adultos de $P$. longifila capturados en las trampas de luz con panel pegante (datos transformados en $\log \mathrm{x}$ ).

\begin{tabular}{lrrrccl}
\hline Fuente de variabilidad & G.L. & S.C. & C.M. & Fc. & Ftab. & Significación \\
\hline Tratamiento & 3 & 0.7701 & 0.2567 & 18.7837 & 3.86 & $* *$ \\
Bloque & 3 & 4.6647 & 1.5549 & 113.7776 & 6.99 & $* *$ \\
Error Experimental & 9 & 0.1230 & 0.0137 & & 3.86 & \\
Total & 15 & 5.5578 & & & &
\end{tabular}

Donde: CV: coeficiente de variabilidad; GL: grados de libertad; SC: suma de cuadrados; CM: cuadrados medios; Fc: F calculado; Ftab: F tabulado. 
Diversas investigaciones sobre trampas luminosas en el Perú y el mundo indican que para la captura de la mayoría de insectos voladores principalmente lepidópteros, coleópteros y dípteros, la luz negra o luz ultravioleta cercana es la más eficaz dentro del espectro electromagnético, pues se asume que la mayoría de los insectos tienen una percepción máxima en la banda del violetaultravioleta; sin embargo, para el caso específico de $P$. longifila esta afirmación no sería del todo cierta, puesto que, según los resultados obtenidos, la mayor atracción de los adultos de $P$. longifila tanto hacia las trampas de luz blanca y negra fluorescentes, estaría demostrando que estos insectos son atraídos hacia un amplio rango de longitudes de onda, que irían desde las porciones de la luz ultravioleta cercana o UV-A hasta la luz visible.

La menor capacidad de atracción de los adultos de $P$. longifila de la trampa de luz amarilla incandescente respecto a las trampas de luz blanca y luz negra (aunque estadísticamente similar a esta última), se debería principalmente a diversos factores, entre ellos al tamaño de la fuente de luz, referido a si es de un tubo fluorescente o una bombilla de luz, al margen del color ya que en ambos casos se da la captura.

Se debe considerar que los adultos de Prodiplosis, se protegen en el día, guareciéndose en lugares sombreados y sin ventilación, como es el caso de barreras físicas o biológicas que se encuentran dentro o fuera del campo, así como en la parte inferior de las plantas o en surcos profundos, como es el caso de la producción de espárrago blanco, o cuando los surcos se encuentran en posición perpendicular a la dirección del viento. En esparragueras inducidas a una cosecha por encima de la capacidad promedio del cultivo la planta empieza a ramear y aperturar filocladios a nivel del suelo y este follaje le sirve al insecto para protegerse, reproducirse e iniciar reinfestaciones. A la atracción de los insectos adultos por las trampas de luz y por el pequeño tamaño que tienen, se añade el hecho de que son transportados con ayuda del viento, sobre todo cuando las infestaciones son altas, permitiendo la captura en el panel pegante (Castillo, 2006).

Las trampas de luz amarilla incandescente tienen una menor densidad de flujo luminoso (iluminación) que la trampa fluorescente de luz blanca, esta

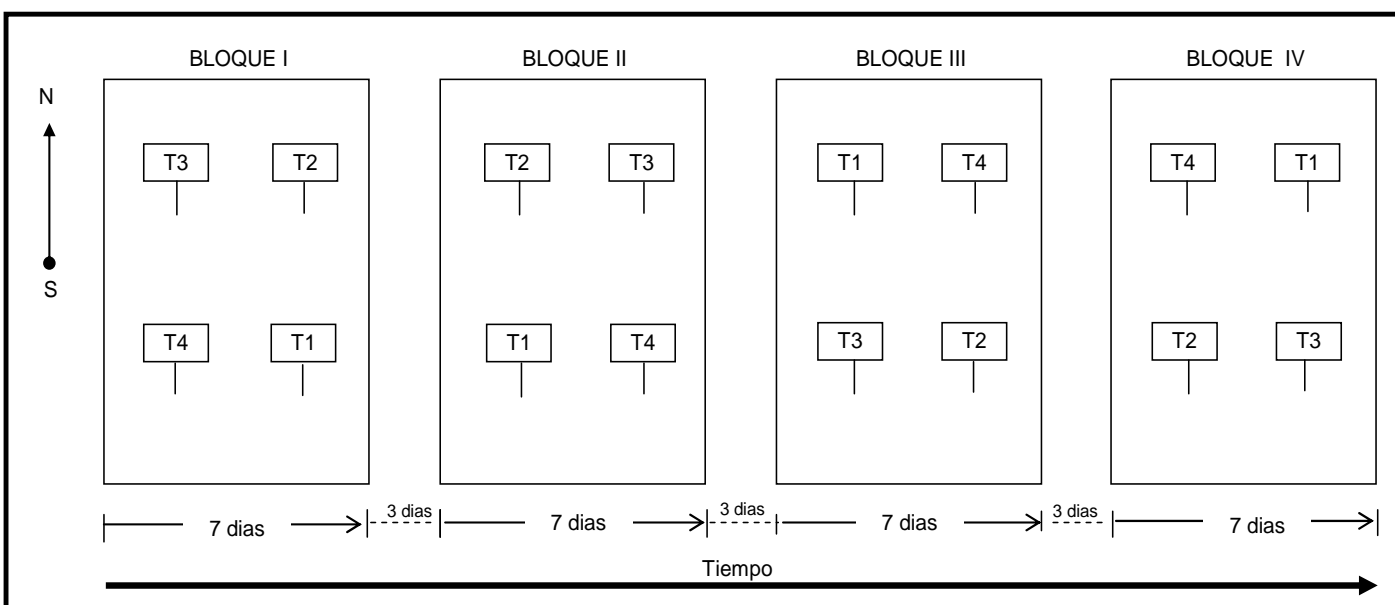

Figura 6. Distribución temporal del experimento.

Leyenda:

T1 Trampa de luz amarilla incandescente

T2 Trampa de luz blanca fluorescente

T3 Trampa de luz negra fluorescente

T4 Trampa de luz amarilla a querosene

Fuente: Elaboración propia (2008). 
característica influiría negativamente en la captura de estos insectos; la longitud de onda de la trampa de luz amarilla incandescente tiene un espectro de emisiones continuo y contiene del 15 al 25 por ciento de la luz visible, la mayoría de la luz radiada está dentro del rango del infrarrojo cercano y se pierde como calor, comparado con la luz blanca y luz negra o ultravioleta cuya mayor radiación se encuentra comprendida entre el rango de la luz visible y la ultravioleta cercana. Según los resultados, este último es el más eficaz para la captura de los adultos de $P$. longifila coincidiendo con Truman (1974), citado por Mazza et al. (2004), Huffaker \& Rabb (1984), Mathews (1984), Nacional Academy of Sciences (1985), Fernández-Rubio (1992) y Vergara (2000).

De acuerdo a lo afirmado por Southwood (1978), Martín \& Woodcook (1983), Nacional Academy of Sciences (1985), Cisneros (1995) y Carranza et al. (1995), se ha podido determinar que la cantidad de energía radiante emitida y la intensidad luminosa, también ejercen una influencia marcada en la captura de los insectos lucípetos, tal es así que la trampa de luz amarilla a querosene presenta aproximadamente 20 veces menos capturas de adultos de $P$. longifila que la trampa de luz amarilla incandescente, posiblemente por ser una fuente de luz de menor tamaño, menor intensidad luminosa y menor energía radiante. El uso de estas lámparas artesanales a querosene u otras formas de combustión, deben descartarse en un programa de control de esta plaga.

Los adultos de $P$. longifila son de hábitos nocturnos, donde se asume que el insecto realiza su apareamiento y reproducción. Es muy común observar en campo que los insectos tienen desplazamientos, que son ayudados por el viento y pueden colonizar campos que se encuentran alejados, incluso en kilómetros, y otro desplazamiento que realiza dentro del campo infestado, a nivel del tercio inferior de la planta al ras del suelo. Los órganos que puede infestar este insecto son los brotes, que se convierten en los futuros tallos; pero en el momento de la floración, el insecto oviposita en la inflorescencia masculina logrando poblaciones a niveles de millones de individuos adultos por hectárea, si los campos se destinan para un segundo brotamiento el insecto infesta el mismo campo, al mismo tiempo, se moviliza a otros campos susceptibles para el ataque de esta plaga. Por ello, el momento de la instalación de las trampas de luz con panel pegante, comprende desde el brotamiento hasta pasada la floración del espárrago. Este parámetro permite el manejo del insecto ya que al reducir sus poblaciones, se reduce también el número de huevos y por consiguiente la infestación, sin usar pesticidas. Fuera de estas etapas fenológicas la presencia del insecto en el cultivo no es relevante, ya que tiene pocos lugares donde poner los huevos y se reproduzca porque la arquitectura de la planta no se lo permite.

\section{Conclusiones.}

1. Las trampas de luz con panel pegante con lámparas de luz blanca y luz negra fluorescentes atraen y capturan con mayor eficiencia un mayor número de adultos de $P$. longifila.

2. La eficiencia de control de las trampas de luz con panel pegante considera la diferencia en el tamaño de la fuente de luz, la diferencias de longitud de onda de cada tipo de luz, como parámetros de atracción y otros factores como la ubicación de la trampa, el estado fenológico del cultivo al instalar la trampa, y las condiciones agroecológicas del cultivo atacado.

\section{Literatura citada.}

Carranza H., Dardon D. \& Salguero V. 1995. Evaluación de trampas de luz para capturar adultos de lepidópteros en brócoli. En: Manejo Integrado del Cultivo de Brócoli. Proyecto MIP-ICTA-CATIE-ARF. Guatemala. pp. 2-15.

Castillo J. 2006. Prodiplosis longifila Gagné en la Irrigación Chavimochic - La Libertad. Arenagro cultivando en el desierto - La Libertad. 2(2): 11 - 19.

Cisneros F. 1995. Control de Plagas Agrícola. Full Print. S.R.L. Lima, Perú. 313 p.

Fernández-Rubio F. 1992. Las trampas de luz automáticas para caza de insectos. Rev. Aragón. Ent. 1(2):79-90.

García M. 2006. Manejo de poblaciones de Prodiplosis longifila. Arenagro cultivando en el desierto - La Libertad 2(2): 24-26.

Gil J. 2008. Diagnóstico y evaluación de plagas insectiles y otros. En: Curso Taller Diagnóstico y Evaluación de Plagas. Tingo María 2004. [en línea]. htpp://www.senasa.gob.pe/intranet/capacitacion/cursos/c urso_tingo_maria/diagnosticoevaluacion_plagas_1.pdf. [consulta: 16 mayo 2008].

Huffaker C. \& Rabb R. 1984. Ecological Entomology. A. Wiley - Interscience. Publication. USA.

Márquez J. 2005. Técnicas de colecta y preservación de insectos. Boletín sociedad entomológica Aragonesa. $\mathrm{N}^{\circ}$ 37. 385-408.

Martin H. \& Woodcock D. 1983. The Scientific Principles of Crop Protection. Seventh Edition. Scotland. 496 p.

Matthews G.A. 1984. Pest Management. Edited by Longman Group Limited. New York. USA. 231 p.

Mazza S., Gimenez M. \& Vitti V. 2004. Captura de lepidópteros plagas del algodonero y otros cultivos del norte santafesino en trampas de luz. Boletín Comunicaciones Científicas y Tecnológicas. Universidad Nacional del Nordeste. Argentina.

Mujica N., Fonseca C., Suarez F., Fabian F., Marchena H. \& Cisneros F. 2000. Reducción del uso de insecticidas en el control de la mosca minadora Liriomyza huidobrensis Blanchard por medio de uso de técnicas etológicas. En: Seminario Internacional de Control Etológico: 25-28 set. de 2000. Libro de resúmenes y programa oficial. Lima, Perú. RAAA. pp. 45-47.

Nacional Academy of Sciences. 1985. Manejo y Control de Plagas de Insectos. Vol. 3. México.

Southwood E. 1978. Ecological methods with particular reference to the study of insect populations. London New York. 524 p.

Vergara R. 2000. Retos y posibilidades del control etológico en la agricultura sostenible. En: Arning \& A. Lizarraga. 
Control Etológico: Uso de feromonas, trampas de colores y luz para el control de plagas en la agricultura sostenible. Lima, Perú. Edit. RAAA.

Walker A. \& Galbreath R. 1979. Collecting insects: a test of four types of lamp. New Zealand Entomologist. Vol. 7 $\mathrm{N}^{\circ} \quad 1 . \quad$ [En línea] htpp://www.ento.org.nz/nzentomologist/free:issues/NZE nto07_1_1979/volume\%207-1-83-85.pdf. [Consulta: 15 mayo 2008].

Weinzierl R., Henn T., Koehler P.G. \& Tucker C. L. 2008. Insects attractants and traps. University of Florida. 1 FAS Extension. [en línea]. htpp://www.edis.ifas.ufl.edu/ino80 [consulta: 5 mayo 2008].

${ }^{1}$ Universidad Nacional Agraria La Molina. Av. La Molina S/N, La Molina - Lima. anfiozu@hotmail.com

2 Docente de la Universidad Nacional Agraria La Molina. Av. La Molina S/N, La Molina - Lima. joracava@lamolina.edu.pe

3 Docente de la Universidad Nacional Agraria La Molina Av. La Molina S/N, La Molina - Lima. srodriguez@lamolina.edu.pe 